\title{
New Dawn Fades: From Imaging to Quantitative Imaging Biomarkers and Beyond
}

\author{
Miloš A. Lučić1-3
}

\section{ABSTRACT}

In the modern ages of rapid technological and computational advances, development of numerous novel diagnostic imaging techniques have enabled not only visual detection of disease processes, but also provided the tools to achieve quantified functional, structural, dynamic, metabolic, and molecular information. A pathway toward the quantitative imaging by use of quantitative imaging biomarkers analysis has been widely opened, enabling further development of radiomics, radiogenomics, and radioproteinomics analysis in addition, and initiating the dawn of theranostics, eventually bringing the wishful personalised medicine concept closer to reality.

Key words: Radiology, Diagnostic Imaging, Quantitative Imaging, Quantitative Imaging Biomarkers, Radiomics, Personalised Medicine.
(1) Medical Faculty, University of Novi Sad, Serbia

(2) Faculty of Medicine, University of Banja Luka, the Republic of Srpska, Bosnia and Herzegovina.

(3) Diagnostic Imaging Centre, Oncology Institute of Vojvodina, Sremska Kamenica / Novi Sad, Serbia.

\section{Correspondence:}

MILOŠ A. LUČIĆ

E:milos.a.lucic@gmail.com

\section{ARTICLE INFO}

Received: 1 June 2019

Accepted: 5 June 2019
Living in the era of an almost unimaginable technological and computational advances in almost all areas of our lives, we have been witnessing a number of extraordinary newly developed imaging techniques and methods. Since the developement of X-rays, ultrasound (US) technique, computerised tomography (CT), and finally magnetic resonance imaging (MRI) emerged, introducing a whole new spectrum of possibilities to produce images and to differentiate between the normal and pathological tissues. However, most of the diagnostic imaging methods and procedures that radiologists are using in a routine practice nowadays were originally developed to detect and diagnose a disease by visualising the signals alterations caused by the underlying disease processes, and hence recognising the pathological pattern, with only a limited attempts to quantify what was seen ${ }^{1}$.

Understanding that the role of diagnostic imaging does not comprise the means to depict anatomical and morphologic abnormalities only, a transitional shift from an entirely morphoanatomically oriented imaging methods towards those that incorporate multimodal or multiparametric acquired data, including functional, structural, dynamic, metabolic, and molecular information ${ }^{2}$ has begun in the last decades and is still and unstopably ongoing, especially intenselly in the field of neuroimaging.

Based on the irrefutable statement of Cha in 2006, that to treat patients with brain tumors without the use of neuroimaging is impossible to imagine ${ }^{2}$, we are already aware that current technological capabilities in neuroimaging have evolved to the level that allows us not only to visualise the tumorous processes in the brain, but also to scrutinise cellular and bioarchitectural alterations of tumorous tissue, to characterise and grade brain tumors, as well to observe and follow-up early and late effects of treatment response in the patients ${ }^{2,3}$.

Recognising and using ex vivo biomarkers (eg, genomic, proteomic, etc) in the clinical practice, a necessity to accelerate the information flow in regard to disease prognosis or outcome emerged, resulting in a particular interest to develop reliable in vivo biomarkers, in a first place non-invasive imaging biomarkers as rapid as possible ${ }^{3}$. 
Therefore, using both new and emerging techniques, but also some already present in clinical practice, a new concept of quantitative imaging has been introduced, enabling a real metric quantification of pathological processes features. According to Quantitative Imaging Biomarkers Alliance (QIBA) of Radiological Society of North America, quantitative imaging represents the extraction of quantifiable features from medical images for the assessment of normal or the severity, degree of change, or status of a disease, injury, or chronic condition relative to normal. Quantitative imaging develops, sstandardises and optimises anatomical, functional and molecular imaging acquisition protocols, data analyses, display methods and reporting structures that permit the validation of accurately and precisely obtained image-derived metrics with anatomically and physiologically relevant parameters, including treatment response and outcome, and the use of such metrics in research and patient care ${ }^{4}$.

Quantitative imaging has already become an increasingly used metric tool in current radiology practice, with the application from research trials and investigations to daily use in radiology reading rooms. Imaging methods that quantify imaging features assist in the clinical assessment of many patients, providing a source of reliable biomarkers for a spectrum of diverse diseases, that are used to provide more accurate diagnosis and/or prognosis, to determine the choice of therapy or to monitor therapy response ${ }^{5}$.

Being based whether on hybrid/molecular imaging techniques, such as positron emission tomography (PET), combined with $\mathrm{CT}_{\text {or }} \mathrm{MRI}^{6}$, or in several cases deriving from CT, many of these outstanding techniques, providing the quantitative imaging biomarkers (QIBs), are actually arrising from MR imaging, which, even after almost forty years of continuous improvements, does not cease to surprise us with the novel achievements and possibilities. Different MRI derived techniques are actually allowing us both to observe the meticulous morphometric details, and to characterise various tissue structures on different magnetic field strength devices, but also to examine the biochemical tissue composition in vivo by use of ${ }^{1} \mathrm{H}$ MR spectroscopy, to obtain dynamic data on tissue perfusion by use of perfusion MRI with exogenous or endogenous contrast tracer, to obtain functional information and to map the brain activities by use of functional MRI and to get the insight into the tissue internal bioarchitecture and brain pathways by use of diffusion imaging, including diffusion tensor and/or kurtosis imaging 5,7.

In addition, capability to collect important metabolic and molecular information by integration of CT or MRI devices in hybrid systems with positron emission tomography (PET) by use of numerous and different specific radionuclide tracers opened the door to the whole new quantitative imaging biomarker (QIB) molecular data sets assessment ${ }^{6,8,9}$.

Based on the information provided, QIBs could be generally categorised as morphoanatomical, dynamic, structural/textural, functional, metabolic and/or molecular, whereas each QIB represents the final result of a predefined image acquisition process, resulting in a quantifiable image from the patient under evaluation, reconstructed by the computer processing algorithm by automated or manual identification of the relevant regions of interest (ROI), measured and reported usually by application of another computer algorithm or arteficial inteligence ${ }^{1}$.

Since a good and qualified biomarker should have three properties, including biological relevance to the disease process under the study, sensitivity to the disease process, and reliability, ie good reproducibility ${ }^{10}$, QIBs are strictly assessed to provide full performance under study conditions, in order to enable better in vivo features quantitative metric evaluation ${ }^{1}$.

Therefore the constant endeavor to develop, validate, and clinically implement both prognostic biologically driven QIBs, that could predict the disease progression probability in the absence of treatment, and predictive QIBs, that may indicate the outcome of the applied therapy is of utmost importance ${ }^{2}$, especially in oncology, and highly valuable in predicting the final disease response, progression-free survival, recurrence and overall survival of the patients ${ }^{11,12}$.

Permanent and continuous introduction of new, underliying disease processes sensitive and reproducible QIBs, almost certainly continues to fulfil a large number of gaps in our heretofore understanding of physiological and pathological processes in the whole human body, causing in- 
evitable shift from quantitative imaging towards radiomics analysis, and finaly, personalised medicine $\mathrm{e}^{13,14}$.

Understanding that diagnostic images are not only images anymore, but real data bases, containing multi-layered, statistically measurable information, a whole new area of unique radiomics possibilities in radiology has opened ${ }^{15}$, firmly directing the future of medicine through the accurate early diagnosis and maximaly personalised and individually tailored therapy treatments to the future concept of personalised and precise medicine ${ }^{12}$.

In the era of radiomics, but also radiogenomics and radioproteinomics data mining out of radiological diagnostic images, relating in vivo

\section{ACKNOWLEDGEMENTS}

Author acknowledge the financial support of the Provincial Secretariat for Science and Technology Development; Grant No. 142-451-2151/2019. quantitative imaging biomarkers to genetic, molecular and biochemical features of the disease or tumour, it is to expect that imaging would became an inevitable and crucial milestone in the patient oriented therapy planning, monitoring and follow-up, by use of non-invasive or minimally invasive treatment options, especially taking into the account the apparent dawn of ther$\operatorname{anostics}^{12,16}$.

Moreover, already developed applications and techniques, such as chemical exchange saturation transfer (CEST) imaging ${ }^{17}$, or MRI fingerprinting ${ }^{18,19}$ could lead us into the nowadays still hardly imaginable directions in the imminent future, with potentially irreversible impact on radiological profession - toward imaging without images.

\section{REFERENCES}

1. Raunig DL, McShane LM, Pennello G, Gatsonis C, Carson PL, Voyvodic JT, et al; QIBA Technical Performance Working Group. Quantitative imaging biomarkers: a review of statistical methods for technical performance assessment. Stat Methods Med Res 2015;24(1):27-67.

2. Cha S. Update on brain tumor imaging: from anatomy to physiology. AJNR Am J Neuroradiol 2006;27(3):47587.

3. Buckler AJ, Paik D, Ouellette M, Danagoulian J, Wernsing G, Suzek BE. A novel knowledge representation framework for the statistical validation of quantitative imaging biomarkers. J Digit Imaging 2013;26(4):61429.

4. Radiological Society of North America (US). Quantitative imaging biomarkers alliance [Internet]. Radiological Society of North America (US); 2019 [Cited 2019 Jun 24]. Available from: https://www.rsna.org/en/research/quantitative-imaging-biomarkers-alliance.

5. Rosenkrantz AB, Mendiratta-Lala M, Bartholmai BJ, Ganeshan D, Abramson RG, Burton KR, et al. Clinical utility of quantitative imaging. Acad Radiol 2015;22(1):33-49.

6. Anderson CJ, Lewis JS. Current status and future challenges for molecular imaging. Philos Trans A Math Phys Eng Sci 2017;375(2107). pii: 20170023. doi: 10.1098/rsta.2017.0023.

7. Martín Noguerol T, Martínez Barbero JP. Advanced dif- fusion MRI and biomarkers in the central nervous system: a new approach. Radiologia 2017;59(4):273-85.

8. Cook GJR, Azad G, Owczarczyk K, Siddique M, Goh V. Challenges and Promises of PET Radiomics. Int J Radiat Oncol Biol Phys 2018;102(4):1083-9.

9. Lee JW, Lee SM. Radiomics in oncological PET/ CT: clinical applications. Nucl Med Mol Imaging 2018;52(3):170-89.

10. Tofts PS, Collins DJ. Multicentre imaging measurements for oncology and in the brain. Br J Radiol 2011;84(Spec No 2):S213-26.

11. Saadatpour Z, Rezaei A, Ebrahimnejad H, Baghaei B, Bjorklund $\mathrm{G}$, Chartrand $\mathrm{M}$, et al. Imaging techniques: new avenues in cancer gene and cell therapy. Cancer Gene Ther 2017;24(1):1-5.

12. European Society of Radiology. Medical imaging in personalised medicine: a white paper of the research committee of the European Society of Radiology (ESR). Insights Imaging 2015;6(2):141-55.

13. Acharya UR, Hagiwara Y, Sudarshan VK, Chan WY, $\mathrm{Ng} \mathrm{KH}$. Towards precision medicine: from quantitative imaging to radiomics. J Zhejiang Univ Sci B 2018;19(1):6-24.

14. Lambin P, Leijenaar RTH, Deist TM, Peerlings J, de Jong EEC, van Timmeren J, et al. Radiomics: the bridge between medical imaging and personalized medicine. Nat Rev Clin Oncol 2017;14(12):749-62. 
15. Gillies RJ, Kinahan PE, Hricak H. Radiomics: images are more than pictures, they are data. Radiology 2016 Feb;278(2):563-77.

16. Lee DY, Li KC. Molecular theranostics: a primer for the imaging professional. Am J Roentgenol 2011;197(2):318-24.

17. Jones KM, Pollard AC, Pagel MD. Clinical applications of chemical exchange saturation transfer (CEST) MRI. J Magn Reson Imaging 2018;47(1):11-27.
18. Ma D, Gulani V, Seiberlich N, Liu K, Sunshine JL, Duerk JL, et al. Magnetic resonance fingerprinting. Nature 2013;495(7440):187-92.

19. Cohen O, Huang S, McMahon MT, Rosen MS, Farrar CT. Rapid and quantitative chemical exchange saturation transfer (CEST) imaging with magnetic resonance fingerprinting (MRF). Magn Reson Med 2018;80(6):2449-63. 